\title{
Sobrevivência e crescimento do pinhão-manso em função do método de aplicação e formulações de hidrogel
}

\author{
João A. L. Dranski ${ }^{1}$, Artur S. Pinto Junior' ${ }^{1}$, Marcelo A. Campagnolo', \\ Ubirajara C. Malavasi ${ }^{2} \&$ Marlene M. Malavasi $^{2}$
}

\begin{abstract}
RESUMO
O trabalho comparou o efeito de formulações e métodos de aplicação de hidrogel no plantio sobre a sobrevivência e crescimento inicial de mudas de pinhão-manso (Jatropha curcas L.). O ensaio foi conduzido em Pato Bragado, PR, durante os anos de 2009 e 2010. O delineamento experimental foi em blocos casualizados, em arranjo fatorial 5 x 2, compreendendo cinco formulações de hidrogel $(0,0 ; 2,5 ; 5,0 ; 7$,5 e $10,0 \mathrm{~g} \mathrm{~L}^{-1}$ ) e dois métodos de aplicação (imersão do sistema radicular em hidrogel previamente hidratado e aplicação de 0,5 L diretamente na cova). As variáveis analisadas incluíram a porcentagem de sobrevivência, o incremento na altura e no diâmetro do coleto e o número de ramificações quantificadas aos três meses e dezoito meses do plantio. Após três meses do plantio a análise revelou efeitos das diferentes formulações, com ajuste linear para sobrevivência, em ambos os métodos de aplicação. Para os incrementos na altura e no diâmetro do coleto houve ajuste polinomial quadrático para a aplicação de $0,5 \mathrm{~L} \mathrm{cova}^{-1}$. Após dezoito meses do plantio não foram observados efeitos significativos ( $p>0,05)$ para os métodos de aplicação ou formulações de hidrogel. $\mathrm{O}$ uso da formulação $7,0 \mathrm{~g} \mathrm{~L}^{-1}$ de hidrogel aplicado diretamente na cova com volume de $0,5 \mathrm{~L} \mathrm{cova}^{-1}$ aumentou o sucesso do estabelecimento do povoamento de pinhão-manso e formulações acima da sugerida reduziram o crescimento das mudas, nas condições do oeste do Paraná.
\end{abstract}

Palavras-chave: Jatropha curcas L., plantio, hidroretentor, dose

\section{Survival and seedling growth of physic nut as a function of method of application and hydrogel formulations}

\begin{abstract}
This study compared the effects of formulations and methods of hydrogel application on the survival and initial seedling growth of physic nut (Jatropha curcas L.). The study was conducted in Pato Bragado, PR during 2009 and 2010. The experimental design was in randomized blocks, arranged in a $5 \times 2$ factorial composed by five formulations of hydrogel $\left(0,2.5,5.0,7.5\right.$, and $\left.10.0 \mathrm{~g} \mathrm{~L}^{-1}\right)$ and two application methods (immersion of roots into hydrogel previously hydrated and application of $0.5 \mathrm{~L}$ directly in the planting hole). Measurements included the percentage of survival, seedling height and diameter increments, and number of branches three and eighteen months after planting. The analysis with three months data revealed significant effects $(p<0.05)$ of the tested hydrogel formulations with a linear adjustment for seedling survival from both application methods. The increments in seedling height and diameter showed a quadratic polynomial response for the application of $0.5 \mathrm{~L}$ of hydrogel per planting hole. After eighteen months, no significant difference $(p>0.05)$ was observed for the application methods or hydrogel formulations. The use of $0.5 \mathrm{~L}$ of a $7.0 \mathrm{~g} \mathrm{~L}^{-1}$ of hydrogel directly into the planting hole favored significantly the establishment of physic nut seedling and formulations above the suggested concentration reduced seedling growth in western Paraná.
\end{abstract}

Key words: Jatropha curcas L., planting, hydrophillic polimer, dose 


\section{INTRODUÇÃO}

Na implantação de povoamentos florestais a disponibilidade de água durante as primeiras semanas após o plantio influi diretamente no estabelecimento e no desenvolvimento das plantas. Em muitos casos, a prática da rega em mudas florestais durante o plantio e após as primeiras semanas tornase necessária para a garantia do sucesso no estabelecimento; contudo, tal prática se torna, em alguns casos, inviável, econômica e operacionalmente ou até mesmo impraticável, em função da disponibilidade de água (Carneiro, 1995; Jhurry, 1997; Buzetto et al., 2002; Malavasi et al., 2005; Thomas, 2008).

O emprego de polímeros hidrorretentores (hidrogeis) emerge alternativamente para minimizar problemas vinculados à deficiência hídrica pós-plantio. Os hidrogeis são produtos de reconhecida capacidade de absorção e adsorção da água gravitacional na rizosfera, para as raízes. Esses polímeros podem, ainda, proteger o sistema radicular contra a desidratação no ato do plantio minimizando custos com a prática de replantio quando o ambiente e a sazonalidade impõem restrições à implantação (Jhurry, 1997; Sarvas, 2003; Vale et al., 2006; Leciejewski, 2009).

A eficiência do uso de hidrogel nos tratos silviculturais de mudas depende do método de aplicação e da formulação com que o polímero é disponibilizado (Jhurry, 1997; Buzetto et al., 2002). Alguns métodos são conhecidos, como a imersão em solução saturada de hidrogel do sistema radicular antevendo o plantio, incorporação do hidrogel não hidratado ao substrato de formação das mudas ou incorporado ao solo. O hidrogel pode, ainda, ser aplicado em solução com diferentes formulações, diretamente na cova ou com o auxílio de plantadeiras manuais (Buzetto et al., 2002; Vallone et al., 2004; Sarvas et al., 2007; Thomas, 2008; Saad et al., 2009).

O pinhão-manso (Jatropha curcas L.) é internacionalmente reconhecido por ser uma espécie promissora para fins energéticos devido à qualidade e à quantidade do óleo obtido das sementes bem como pelas propriedades medicinais e biocidas (Gubitz et al., 1999). A cultura não possui um sistema de produção validado para a região oeste do Paraná que garanta o sucesso na implantação da cultura; por outro lado, são poucas as informações do uso de hidrogel para a cultura do pinhão-manso. Adicionalmente não existe, na região oeste do Paraná, cultivo comercial da cultura e os esforços se centram em pesquisas agronômicas no tocante ao melhoramento cultural da espécie, com o intuito de torná-la viável economicamente em termos de produtividade. Diante do exposto o objetivo do presente trabalho foi comparar o efeito de formulações e métodos de aplicação de hidrogel na ocasião do plantio de mudas de pinhão-manso (Jatropha curcas L.) sobre a sobrevivência e crescimento pós-plantio.

\section{Material e Métodos}

O experimento foi conduzido nos anos de 2009 e 2010, na Estação Experimental de Pato Bragado, PR, com coordenadas geográficas de $24^{\circ} 39^{\prime \prime} 43^{\prime} \mathrm{S}$ e $54^{\circ} 15^{\prime \prime} 53^{\prime} \mathrm{W}$ e altitude de $247 \mathrm{~m}$. O clima da região, segundo classificação de Köppen, é subtropical úmido mesotérmico com verões quentes (temperatura média superior a $22{ }^{\circ} \mathrm{C}$ ) com tendência à concentração de chuvas e invernos com geadas pouco frequentes (temperatura média inferior a $18^{\circ} \mathrm{C}$ ) e precipitação média anual de $1.500 \mathrm{~mm}$. Os dados agroclimáticos do período avaliativo estão grafados na Figura 1.

O solo da área experimental é classificado como Latossolo Vermelho distrófico típico ( $\mathrm{LVd}$ ), de textura argilosa (EMBRAPA, 2006) com os respectivos atributos químicos: matéria orgânica $=18,57 \mathrm{~g} \mathrm{dm}^{-3} ; \mathrm{P}=4,74 \mathrm{mg} \mathrm{dm}^{-3} ; \mathrm{K}^{+}=0,43$ $\mathrm{cmol}_{\mathrm{c}} \mathrm{dm}^{-3} ; \mathrm{Mg}^{2+}=1,21 \mathrm{cmol}_{\mathrm{c}} \mathrm{dm}^{-3} ; \mathrm{Ca}^{2+}=2,46 \mathrm{cmol}_{\mathrm{c}} \mathrm{dm}^{-3} ;$ $\mathrm{Al}^{3+}=0,40 \mathrm{cmol}_{\mathrm{c}} \mathrm{dm}^{-3} ; \mathrm{H}+\mathrm{Al}=5,83 \mathrm{cmol}_{\mathrm{c}} \mathrm{dm}^{-3} ; \mathrm{Cu}=15,62$ $\mathrm{mg} \mathrm{dm}{ }^{-3} ; \mathrm{Zn}=1,88 \mathrm{mg} \mathrm{dm}^{-3} ; \mathrm{Mn}=179 \mathrm{mg} \mathrm{dm}^{-3} ; \mathrm{Fe}=66,25$ $\mathrm{mg} \mathrm{dm}{ }^{-3} ; \mathrm{CTC}=9,38 \mathrm{cmol}_{\mathrm{c}} \mathrm{dm}^{-3} ;$ Saturação de bases $=41,5 \%$; Saturação por alumínio $=9,3 \% \mathrm{e} \mathrm{pH} \mathrm{em} \mathrm{CaCl}_{2}=4,46$.

O delineamento experimental foi em blocos ao acaso, em arranjo fatorial $5 \times 2$, compreendendo cinco formulações de hidrogel $\left(0,0 ; 2,5 ; 5,0 ; 7,5\right.$ e $\left.10,0 \mathrm{~g} \mathrm{~L}^{-1}\right)$ e dois métodos de aplicação no plantio (imersão do sistema radicular em hidrogel previamente hidratado e aplicação via solução diretamente na cova com volume de $\left.0,5 \mathrm{~L}_{\text {cova }}{ }^{-1}\right)$ conforme sugerido por Thomas (2008) e Buzetto et al. (2002) respectivamente, com cinco repetições. A unidade experimental foi composta por quatro mudas.

O método da imersão do sistema radicular foi efetuado por meio da imersão do torrão radicular durante dois minutos, em hidrogel acondicionado em recipientes com capacidade de 1 litro, preenchidos com as devidas formulações. Para o método da aplicação do hidrogel diretamente na cova a formulação e o respectivo volume foram alocados na base da cova; após a aplicação do polímero para ambos os métodos, ocorreu o plantio propriamente dito.

Para a produção de mudas foram utilizadas sementes provenientes de Pedro Juan Caballero, PY cedidas pela Rural Biodiesel Ltda., sendo semeadas diretamente em tubetes de $120 \mathrm{~cm}^{3}$ com seção circular e seis estrias, preenchidos com substrato comercial à base de casca de pinus e acomodados em suportes plásticos com capacidade de ocupação para 96 tubetes. Empregaram-se $40 \mathrm{mg}$ de N, $\mathrm{P}_{2} \mathrm{O}_{5}$ e $\mathrm{K}_{2} \mathrm{O}$ por muda, sendo todo o conteúdo do fósforo incorporado ao substrato com auxílio de betoneira e o conteúdo de nitrogênio e potássio disponibilizado pela água de irrigação em três aplicações quinzenais após a emergência das plântulas (Gonçalves et al., 2000; Paulino et al., 2011).

O tempo de formação das mudas foi de 60 dias após a emergência, em ambiente de casa de vegetação com $50 \%$ de sombreamento. As mudas foram irrigadas por 45 dias e o substrato mantido próximo à capacidade de saturação. Os últimos 15 dias de formação foram destinados à rustificação, através da redução gradativa do regime de regas e redução pela metade da ocupação das mudas nos suportes plásticos, por meio do intercalamento entre células ocupadas pelas mudas e sem ocupação, resultando em 46 mudas por suporte.

Mudas foram transplantadas ao campo quando apresentaram, em média, $19,7 \mathrm{~cm}$ de altura, $8,07 \mathrm{~mm}$ de diâmetro do coleto e 4 folhas, em fevereiro de 2009, com espaçamento de 3,0 x 2,0 m. A dimensão da cova foi, em média, de $0,10 \mathrm{~m}$ de raio por $0,40 \mathrm{~m}$ de profundidade. Os tratos culturais envolveram a roçada de plantas espontâneas e coroamento das mudas num 
raio de $0,60 \mathrm{~m}$. Não houve correção dos níveis de fertilidade do solo tendo em vista que no cultivo do pinhão-manso são incipientes as informações sobre correção da acidez e fertilidade dos solos e escassas para Latossolo Vermelho distrófico típico, característico da região oeste do Paraná.

As mensurações incluíram a porcentagem de sobrevivência e o crescimento pós-plantio pelos incrementos na altura da parte aérea $(\mathrm{cm})$ e diâmetro do coleto $(\mathrm{mm})$ tal como pela contagem do número de ramificações primárias aos três e dezoito meses após o plantio.

Os dados obtidos foram averiguados quanto à normalidade da distribuição dos resíduos pelo teste de Lilliefors e homogeneidade da variância pelo teste de Cochran \& Bartlet, e submetidos à análise de variância com o auxílio do software SAEG (ver. 9.0). Quando da existência de diferenças significativas as médias entre os métodos de aplicação foram comparadas pelo teste de Tukey a 0,05 de probabilidade enquanto formulações de hidrogel o foram pela análise de regressão.

\section{Resultados E Discussão}

Os resultados agroclimáticos (Figura 1) indicam que as médias de temperatura se mantiveram em consonância com as normais climatológicas para a região de plantio; contudo, a precipitação acumulada aos três meses após o plantio foi de $185 \mathrm{~mm}$ abaixo da normal climatológica, que se situa em torno de $500 \mathrm{~mm}$. Após este período não se registraram anomalias para a recipitação. Adicionalmente, o Estado do Paraná não apresenta uma estação seca definida nem, tampouco, baixa probabilidade de estiagem por mais de 30 dias. O estado externa ampla variabilidade na distribuição temporal e espacial da precipitação com probabilidade de até $80 \%$ para ocorrência de períodos secos (veranicos) com 15 dias de duração, comumente observados na época do plantio de mudas de espécies lenhosas (Bernardes et al., 1988).

Os resultados acusaram interação significativa para porcentagem de sobrevivência de mudas de pinhão-manso em função dos métodos de aplicação e das formulações de hidrogel aos três meses do plantio $(\mathrm{p}<0,05)$. A imersão do sistema radicular em hidrogel promoveu menor sobrevivência $(65 \%)$ em contraste com a aplicação diretamente na cova $(85,0 \%)$ provavelmente resultado do menor volume de hidrogel por muda.

Houve efeito linear significativo $(p<0,05)$ para sobrevivência de mudas em função das formulações (Figura 2). Mudas com o sistema radicular imerso em hidrogel ou através da aplicação de $10 \mathrm{~g} \mathrm{~L}^{-1}$ diretamente na cova, apresentaram sobrevivência de 83 e $99 \%$, respectivamente, ou seja, um acréscimo de $28 \%$ quando comparado com a testemunha. O uso do hidrogel no ato do plantio de mudas de pinhão-manso sustenta a hipótese do armazenamento e disponibilidade de água no solo nas condições experimentais testadas tendo em vista que neste período houve déficit na precipitação na região de plantio, como relatado na Figura 1. Esta resposta está atrelada aos efeitos do polímero hidrorretentor em prolongar a umidade na rizosfera e minimizar os efeitos da desidratação do sistema radicular das mudas no ato do plantio (Sarvas, 2003; Cortéz et al., 2007).

Em paralelismo com a literatura, os resultados obtidos se assemelham aos verificados por Buzetto et al. (2002) ao relatarem diferenças significativas na sobrevivência de mudas de Eucalyptus urophilla quando da adição de $0,8 \mathrm{~L}^{-1}$

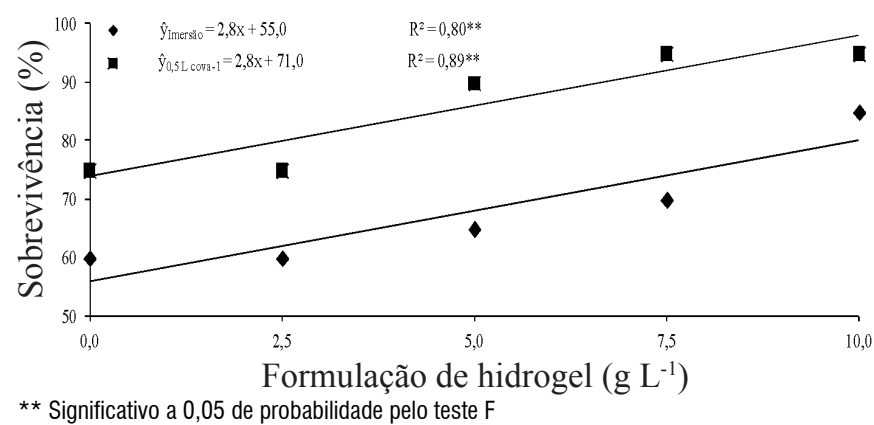

Figura 2. Porcentagem de sobrevivência de mudas de pinhão-manso três meses após o plantio, em função do método de aplicação de hidrogel

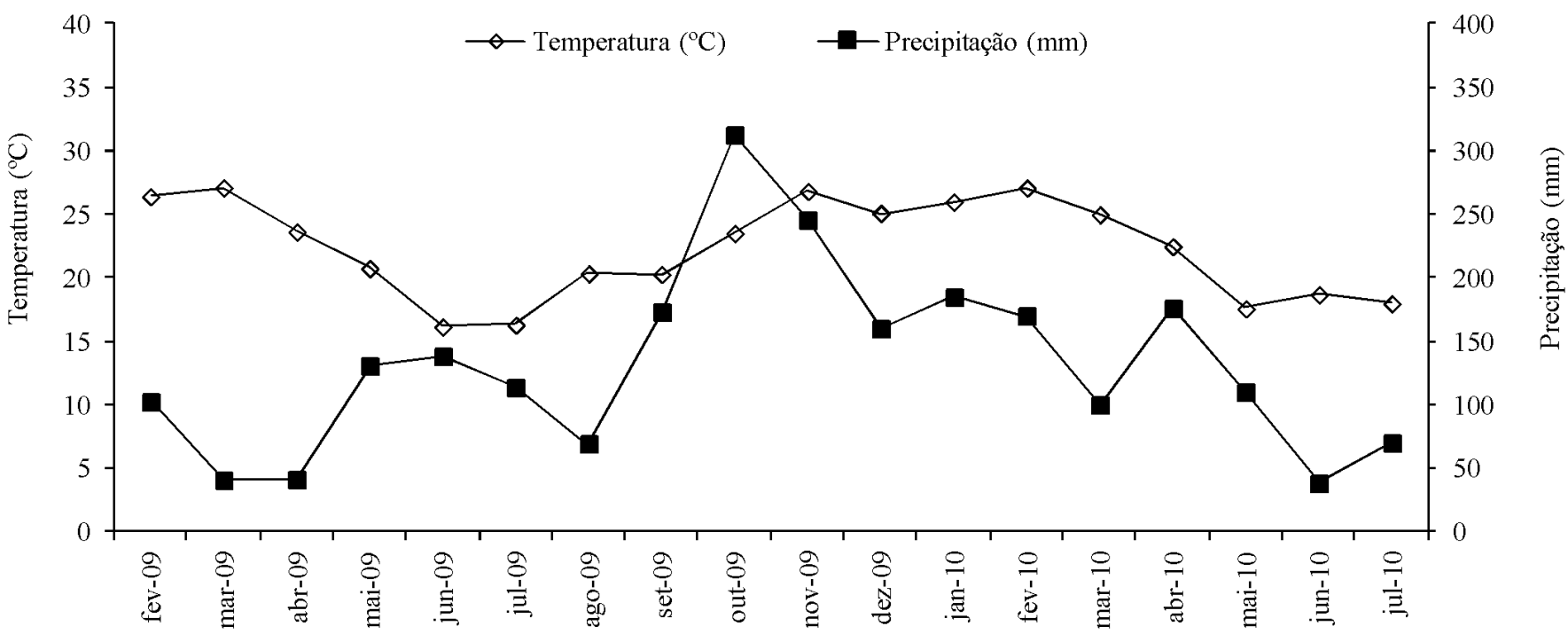

Figura 1. Temperatura média e precipitação acumulada na Estação Experimental de Pato Bragado, PR, entre fevereiro de 2009 a novembro de 2010 
da formulação de $2 \mathrm{~g} \mathrm{~L}^{-1}$ aplicada na base das covas. Thomas (2008) observou aumento de 14 e 6\% na sobrevivência de mudas de Eucalyptus pilularis e Corymbia citriodora subsp. Variegata, respectivamente, para mudas com torrão radicular imerso em hidrogel, com formulação de $2,5 \mathrm{~g} \mathrm{~L}^{-1}$. Para Huttermann et al. (1999), o aumento na sobrevivência de $49 \%$ (controle) para $82 \%$ quando da adição de $0,4 \%$ de hidrorretentor incorporado ao solo, antevendo o plantio de mudas de Pinus halepensis, ocorreu pelo fato do hidrogel reter maior quantidade de água e elevar de 16 para 75 dias a redução de perdas do conteúdo de água no solo. Lopes et al. (2010) observaram que o uso de $0,96 \mathrm{~g}$ de polímero hidratado na cova possibilitou a manutenção do potencial hídrico das mudas de Eucalyptus urograndis em até 37 dias de restrição hídrica, sem comprometer o crescimento.

Após três meses do plantio os resultados da análise de variância apontaram interação significativa $(\mathrm{p}<0,05)$ para os incrementos, na altura e no diâmetro do coleto. Mudas que receberam $0,5 \mathrm{~L}$ de hidrogel na cova externaram maiores médias (19,26 cm e 29,04 mm, respectivamente) em relação às mudas com sistema radicular imerso em hidrogel $(25,18 \mathrm{~cm}$ e 16,69 $\mathrm{mm}$, respectivamente).

No desdobramento da interação entre formulações e métodos de aplicação sobre o crescimento aéreo não houve efeito significativo do aumento das formulações sobre os incrementos na altura e no diâmetro do coleto em mudas com o sistema radicular imerso em hidrogel ( $\mathrm{p}>0,05)$; apesar disto, mudas que receberam $0,5 \mathrm{~L}$ de hidrogel na cova externaram efeito significativo (Figura 3), com ajuste polinomial quadrático $(\mathrm{p}<0,05)$ sendo a formulação de $6,3 \mathrm{~g} \mathrm{~L}^{-1}$ a que resultou no maior incremento na altura $(32,81 \mathrm{~cm})$. Resposta semelhante foi observada no incremento em diâmetro do coleto e maiores médias foram mensuradas em mudas que receberam a formulação de $6,7 \mathrm{~g} \mathrm{~L}^{-1}$, correspondendo a 21,37 $\mathrm{mm}$ com manejo do polímero compreendido pela adição de $0,5 \mathrm{~L}$ na cova.

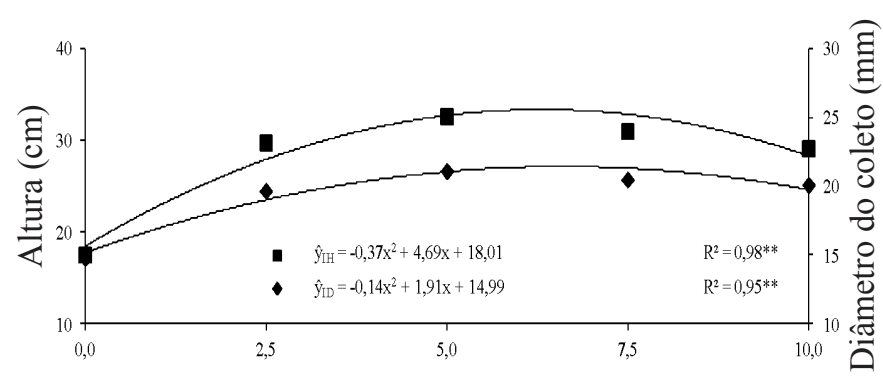

Formulação de hidrogel $\left(\mathrm{g} \mathrm{L}^{-1}\right)$

** Significativo a 0,05 de probabilidade pelo teste $\mathrm{F}$

Figura 3. Incremento na altura $(\mathrm{IH})$ e no diâmetro do coleto (ID) três meses após o plantio de mudas de pinhão-manso submetidas à aplicação de hidrogel, diretamente na cova, com volume de $0,5 \mathrm{~L} \mathrm{cova}^{-1}$

A redução no incremento em altura e no diâmetro do coleto de mudas expostas a formulações maiores que $7 \mathrm{~g} \mathrm{~L}^{-1}$ quando da aplicação de $0,5 \mathrm{~L} \mathrm{cova}^{-1}$, pode estar associada aos efeitos do polímero hidrorretentor nas propriedades físicas e hidráulicas do solo tendo em vista que a expansão do polímero altera a porosidade do solo, diminui o movimento da solução ao elevar a capilaridade e minimiza a relação água/ar, conforme argumentação de Martyn \& Szot (2001), Vallone et al. (2004) e Albuquerque Filho et al. (2009).

$\mathrm{O}$ número de ramificações aumentou linearmente em função do aumento da formulação após três meses do plantio, independentemente do método de aplicação de hidrogel (Figura 4). Mudas sem a adição de hidrogel apresentaram, em média, 2,06 e 1,97 ramificações por muda, em contraste com mudas submetidas à formulação de $10 \mathrm{~g} \mathrm{~L}^{-1}$, que externaram 3,03 e 3,14 ramificações nas mudas com o sistema radicular imerso em hidrogel e aplicação via solução diretamente na cova, respectivamente.

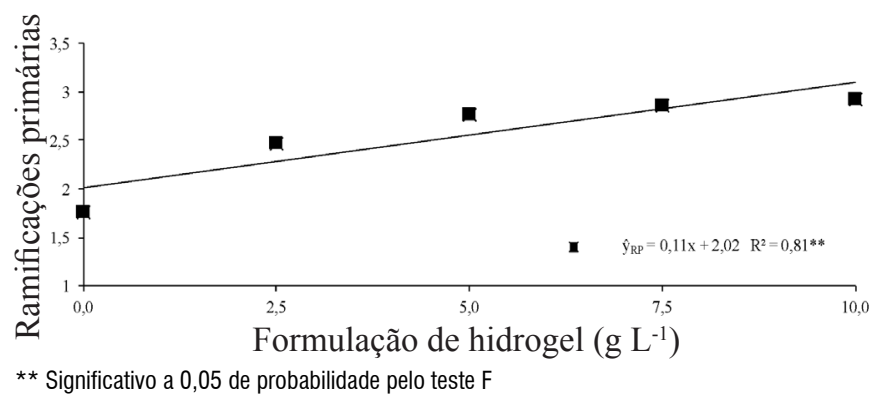

Figura 4. Número de ramificações primárias (RP) em mudas de pinhão-manso três meses após o plantio submetidas a diferentes formulações de hidrogel

Há relatos, na literatura, de que a permeabilidade dos solos diminui progressivamente com o aumento na concentração de polímeros, tanto em solos argilosos quanto arenosos, sugerindo uma limitação no uso desses polímeros quanto ao aspecto da infiltração da água no solo, como reportado por Balena (1998) em um Latossolo Vermelho Escuro (LE) de textura argilosa e em areia marinha do litoral paranaense, bem como por Coelho et al. (2008) cuja condutividade hidráulica em três solos de diferentes texturas (Neossolo Quartzarênico, Latossolo Amarelo e Neossolo Flúvico) reduziu linearmente quando submetidos a formulações crescentes de um polímero hidrorretentor.

O estado higroscópico da muda tem sido apontado como importante fator na sobrevivência após o plantio (Mckay \& White, 1997). A tolerância à dessecação envolve diversos fatores, entre os quais a estabilidade da membrana celular é um requerimento básico para manutenção das funções fisiológicas em vegetais (Bewley, 1979).

Os efeitos do hidrogel na fisiologia de espécies lenhosas apresentam controvérsias. Enquanto Apostol et al. (2009) reportaram inexistir efeitos benéficos no crescimento e nas trocas gasosas em mudas submetidas ao estresse hídrico, Arbona et al. (2005) concluíram que a presença do hidrogel contribuiu não só para a sobrevivência como resultou em maior crescimento e fotossíntese em plantas cítricas.

Após dezoito meses do plantio não houve alteração na porcentagem de sobrevivência cujos valores se mantiveram iguais aos obtidos nas mensurações com três meses do plantio. Para os parâmetros de crescimento quantificados não foram detectadas diferenças significativas $(p>0,05)$ frente aos métodos de aplicação e formulações de hidrogel. Mudas de pinhão-manso externaram médias de $66,04 \mathrm{~cm}$ para o incremento na altura, $16,84 \mathrm{~mm}$ para o incremento no diâmetro 
do coleto e 6,7 ramos primários. Comportamento semelhante foi relatado por Buzetto et al. (2002) em mudas de Eucalyptus urophylla aos nove meses após o plantio, por Vallone et al. (2004) e Vale et al. (2006) na formação e crescimento inicial de mudas de café e por Sarvas et al. (2007) em Pinus sylvestris, após três períodos vegetativos.

A falta de diferenças significativas no crescimento das mudas após o período de dezoito meses do plantio pode estar atrelada ao processo natural de degradação do hidrogel. Segundo Azevedo et al. (2002) e Lopes et al. (2010) a degradação do polímero é potencializada pelo nível de fertilidade do solo em função da concentração de sais contidos nos solos. Desta forma, os altos níveis de alumínio evidenciados na análise química do solo podem ter contribuído para a degradação do polímero hidrorretentor.

Com base nos resultados alcançados o uso do hidrogel aplicado diretamente na cova potencializou o crescimento e a sobrevivência nos primeiros três meses após o plantio, tendo em vista que aos dezoito meses do plantio não houve diferenças significativas na velocidade de crescimento nem na sobrevivência, ficando evidente a atenuação dos efeitos prejudiciais do choque do plantio sobre a mortalidade e o crescimento.

\section{CONCLUSÃO}

O aumento na sobrevivência pós-plantio e a inexistência de efeitos negativos sobre o crescimento a posteriori, sugerem o uso da formulação de até 7,0 $\mathrm{g} \mathrm{L}^{-1}$ de hidrogel diretamente na cova em volume de $0,5 \mathrm{~L} \mathrm{cova}^{-1}$ por ocasião do plantio de mudas de pinhão-manso visando ao estabelecimento da cultura nas condições do oeste do Paraná.

\section{Agradecimentos}

Externamos nossos agradecimentos à Rural Biodiesel Ltda., pelo fornecimento de sementes de Jatropha curcas L.; à CAPES, pela concessão de bolsas e ao $\mathrm{CNPq}$, pelo apoio financeiro ao projeto.

\section{Literatura Citada}

Albuquerque Filho, J. A. C.; Lima, V. L. A. de; Menezes, D.; Azevedo, C. A. V. de; Dantas Neto, J.; Silva Júnior, J. C. Características vegetativas do coentro submetido a doses do polímero hidroabsorvente e lâminas de irrigação. Revista Brasileira de Engenharia Agrícola e Ambiental, v.13, p. 671-679, 2009.

Apostol, K. G.; Jacobs, D. F.; Dumroese, K. Root desiccation and drought stress responses of bareroot Quercus rubra seedlings treated with hydrophilic polymer root dip. Plant and Soil, v.315, p.229-240, 2009.

Arbona, V.; Iglesias, D. J.; Jacas, J.; Primo-Millo, E.; Talon, M.; Cadenas, A. G. Hydrogel substrate amendment alleviates drought effects on young citrus plants. Plant and Soil, v.270, p.73-82, 2005.

Azevedo, T. L. F.; Bertonha, A.; Freitas, P. S. L.; Gonçalves, A. C. A.; Rezende, R.; Dallacort, R.; Bertonha, L. C. Níveis de polímero superabsorvente, freqüências de irrigação e crescimento de mudas de café. Acta Scientiarum, v.24, p.1239-1243, 2002.
Balena, S. P. Efeito de polímeros hidroretentores nas propriedades físicas e hidráulicas de dois meios porosos. Curitiba: UFPR, 1998. 57p. Dissertação Mestrado

Bernardes, L. R. M.; Aguilar, A. P.; Abe, S. Frequência de ocorrência de veranicos no estado do Paraná. Boletim de Geografia, v.6, p.83-108, 1988.

Bewley, J. D. Physiological aspects of desiccation tolerance. Annual Review of Plant Physiology, v.30, p.195-238, 1979.

Buzetto, F. A.; Bizon, J. M. C.; Seixas, F. Avaliação de um polímero adsorvente a base de acrilamida no fornecimento de água no fornecimento de água em mudas de Eucalyptus Urophylla em pós-plantio. Piracicaba: IPEF, 2002. 8p. Circular técnica, 195

Carneiro, J. G. de A. Produção e controle de qualidade de mudas florestais. Curitiba: UFPR/UENF/FUPEF, 1995. 451p.

Coelho, J. B. M.; Barros, M. F. C.; Correa, M. M.; Wanderley, R. A.; Coelho Júnior, J. M.; Figueredo, J. L. C. Efeito do polímero hidratassolo sobre propriedades físico-hídricas de três solos. Revista Brasileira de Ciências Agrárias, v.3, p.253-259, 2008.

Cortés, A. B.; Ramírez, I. X. B.; Eslava, L. F. B.; Niño, G. R. Evaluación de hidrogeles para aplicaciones agroforestales. Revista Ingeniería e Investigación, v.27, p.35-44, 2007.

EMBRAPA - Empresa Brasileira de Pesquisa Agropecuária. Centro Nacional de Pesquisa de Solos. Sistema Brasileiro de Classificação de Solos. 2.ed. Rio de Janeiro: EMBRAPA, 2006. 306p.

Gonçalves, J. L. M.; Santarelli, E. G.; Neto, S. P. M.; Manara, M. P. Produção de mudas de espécies nativas: substrato, nutrição, sombreamento e fertilização. In: Gonçalves J. L. M.; Benedetti, V. Nutrição e fertilização florestal. Piracicaba: IPEF, 2000. Cap.11, p.80-102.

Gubitz, G. M.; Mittelbach, M.; Trabi, M. Exploitation of the tropical oil seed plant Jatropha curcas L., Bioresource Technology, v.67, p.73-82, 1999.

Huttermann, A.; Zommorodi, M.; Reise, K. Addition of hidrogels to soil for prolonging the survival of Pinus Halepensis seedlings subjected to drought. Soil \& Tillage Research, v.50, p.295-304, 1999.

Jhurry, D. Agricultural polymers. Mauritius: Food and agricultural research council, Réduit, AMAS, 1997. 113p.

Leciejewski, P. The effect of hydrogel additives on the water retention curve of sandy soil from forest nursery in Julinek. Journal Water Land Development, v.13a, p.239-247, 2009.

Lopes, J. L. W.; Silva, M. R.; Saad, J. C. C.; Angélico, T. S. Uso de hidrogel na sobrevivência de mudas de Eucalyptus urograndis produzidas com diferentes substratos e manejos hídricos. Ciência Florestal, v.20, p.217-224, 2010.

Malavasi, U. C.; Gasparino, D.; Malavasi, M. M. Semeadura direta na recomposição vegetal de áreas ciliares: efeitos da sazonalidade, uso do solo, exclusão da predação, e profundidade na sobrevivência inicial. Semina, v.26, p.449-454, 2005.

Mckay, H. M.; White, I. M. S. Fine root electrolyte leakage and moisture content: indices of Sitka spruce and Douglas-fir seedling performance after desiccation. New Forests, v.13, p.139-162. 1997.

Martyn, W.; Szot, P. Influence of superabsorbents on the physical properties of horticultural substrates. International Agrophysics, v.15, p.87-94, 2001. 
Paulino, J.; Folegatti, M. V.; Flumignan, D. L.; Zolin, C. A.; Barboza Júnior, C. R. A.; Piedade, S. M. S. Crescimento e qualidade de mudas de pinhão-manso produzidas em ambiente protegido. Revista Brasileira de Engenharia Agrícola e Ambiental, v.15, p.37-46, 2011.

Saad, J. C. C.; Lopes, J. L. W.; Santos, T. A. Manejo hídrico em viveiro e uso de hidrogel na sobrevivência pós--plantio de Eucalyptus urograndis em dois solos diferentes. Engenharia Agrícola, v.29, p.404-411, 2009.

Sarvas, M. Effect of desiccation on the root system of Norway spruce (Picea abies [L.] Karst.) Seedlings and

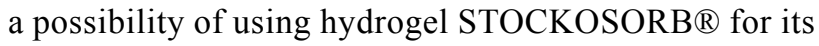
protection. Journal of Forest Science, v.11, p.531-536, 2003.
Sarvas, M.; Pavlenda, P.; Takácov, E. Effect of hydrogel application on survival and growth of pine seedlings in reclamations. Journal of Forest Science, v.5, p.204-209, 2007.

Thomas, D. S. Hydrogel applied to the root plug of subtropical eucalypt seedlings halves transplant death following planting. Forest Ecology and Management, v.255, p.1305-1314, 2008.

Vale, G. R. F.; Carvalho, S. P.; Paiva, L. C. Avaliação da eficiência de polímeros hidroretentores no desenvolvimento do cafeeiro em pós-plantio. Coffee Science, v.1, p.7-13, 2006.

Vallone, H. S.; Guimarães, R. J.; Souza, C. A. S.; Carvalho, J. A.; Ferreira, R. S.; Oliveira, S. Substituição do substrato comercial por casca de arroz carbonizada para produção de mudas de cafeeiro em tubetes na presença de hidrorretentor. Ciência e Agrotecnologia, v.28, p.593-599, 2004. 